Voix et Images

voixetimages

\title{
« D’hier à aujourd'hui, métamorphoses »
}

\section{Isabelle Boisclair}

Volume 37, numéro 1 (109), automne 2011

URI : https://id.erudit.org/iderudit/1006471ar

DOI : https://doi.org/10.7202/1006471ar

Aller au sommaire du numéro

Éditeur(s)

Université du Québec à Montréal

ISSN

0318-9201 (imprimé)

1705-933X (numérique)

Découvrir la revue

Citer ce compte rendu

Boisclair, I. (2011). Compte rendu de [" D’hier à aujourd'hui, métamorphoses »].

Voix et Images, 37(1), 155-160. https://doi.org/10.7202/1006471ar d'utilisation que vous pouvez consulter en ligne.

https://apropos.erudit.org/fr/usagers/politique-dutilisation/ 


\section{F É M I N I S M E S}

"D'hier à aujourd'hui, métamorphoses "

$$
++
$$

ISABELLE BOISCLAIR

Université de Sherbrooke

Si l'on schématise à l'extrême, on peut dire que ce sont d'abord les représentations féminines, surtout dans les œuvres écrites par des hommes, qui retinrent l'attention de la critique féministe, avant que l'on se tourne vers les textes de femmes, à l'affût des jeux d'écriture. En évoluant, les recherches féministes reviennent sur certains points précis, en même temps qu'elles élargissent les champs d'investigation. Ici, l'attention se porte sur l'écriture des femmes dans un genre particulier; là, les recherches, après avoir envisagé le masculin à côté du féminin, embrassent les postures qui y sont liées. Représentatif du premier de ces mouvements, L'annuaire théâtral présente un dossier sur les femmes dans l'institution théâtrale: «Une dramaturgie à soi : l'écriture du thêâtre des femmes au Québec ${ }^{1} »$; issu du second mouvement, un essai de Lori Saint-Martin, Au-delà du nom. La question du père dans la littérature québécoise actuelle $^{2}$, se consacre à la figure du père au sein d'un corpus mixte. Ces démarches se complètent: il s'agit, dans un cas comme dans l'autre, de questionner la socioculture qui nous fait, et la place qu'elle réserve au féminin et au masculin.

L'annuaire théâtral propose un dossier sur le théâtre des femmes au Québec (entre 1975 et 1995), publié sous la direction de Gilbert David. Outre le texte de présentation de David, cinq articles composent le dossier. Trois d'entre eux portent sur une œuvre spécifique : Louise Forsyth s'intéresse au caractère expérimental de l'écriture de La nef des sorcières ${ }^{3}$ (1976); Stéphanie Nutting, à une facette particulière du dispositif énonciatif dans Billy Strauss (1991) de Lise Vaillancourt; et Yves Jubinville, à la réception critique des Fées ont soif (1978) de Denise Boucher. Les articles de Jane Moss et de Gilbert David, pour leur part, envisagent plus largement la question au principe du dossier: Moss se penche sur le problème du corps tandis que David souligne la présence importante de la comédie dans le corpus du thêâtre au féminin et s'attarde à quelques pièces.

1 L'annuaire théâtral, no 46, automne 2009, p. 5-212. 2 Lori Saint-Martin, Au-delà du nom. La question du père dans la littérature québécoise actuelle, Montréal, Presses de l’Université de Montréal, 2010, 428 p. 3 La pièce a été créée par un collectif formé de Marie-Claire Blais, Marthe Blackburn, Nicole Brossard, Odette Gagnon, Luce Guilbeault, Pol Pelletier et France Théoret le 5 mars 1976 au Thêâtre du Nouveau Monde. 
Ce qui étonne au premier chef, sans doute, c'est le rappel que font Jubinville et Forsyth de la réception difficile des deux textes phares que sont La nef des sorcières et Les fées ont soif. Si ces pièces sont aujourd'hui intégrées au canon du théâtre féministe québécois, elles ont d'abord été disqualifiées par maints critiques, sous le prétexte de leur manque de théâtralité ${ }^{4}$, comme si le monologue ne suffisait pas, comme s'il n'était pas une forme délibérément utilisée pour signifier quelque chose - l'isolement des femmes à la veille de la «révolution»- - comme s'il ne constituait pas en lui-même une forme spécifique de l'écriture dramatique. Or, au sujet de ces diverses perceptions, il n'est pas superflu de rappeler ces propos de Françoise Collin, selon qui il y a

une loi de la (bonne) transgression et une institution de l'avant-garde avec ses prêtres, ses gardiens. La littérature française en particulier répète volontiers dans la novation ce qu'elle connaît dans la tradition. Ainsi toute transgression, toute déviance n'est-elle pas recevable. Et on peut se demander si les transgressions imputables aux femmes ne sont pas ignorées ou refoulées par le fonctionnement de l'institution: perçues comme inaptitude à la loi plutôt que comme son surmontement ${ }^{5}$.

L'étude de Jubinville sur la réception critique des Fées ont soif cautionne cette analyse ; tout au moins elle rappelle que ce phénomène a existé, un temps - au moment où se faisaient entendre ces voix de femmes qui, visiblement, ne parlaient plus comme avant, exprimant aussi bien le ressentiment que la revendication. Moment de rupture, donc, qui déstabilisa certainement les récepteurs, nourris jusque-là de la seule perspective masculine sur les femmes. Plus personne aujourd'hui ne songerait à remettre en question la théâtralité de la pièce de Boucher - surtout pas sous prétexte qu'il y a peu d'intrigue. Or, l'étude de Forsyth à propos de La nef des sorcières le souligne, la réception de ces pièces au moment de leur création a fait défaut: «La plupart des critiques adoptèrent une attitude très négative. L'essentiel de leurs reproches, de nature surtout idéologique, se dissimulait souvent derrière une version de la déclaration péremptoire suivante: le spectacle n'était pas du théâtre» (36). La dramaturgie contemporaine valide a posteriori, d'une certaine façon, les explorations menées par les femmes à cette époque. Mais l'examen de leur réception immédiate montre que, de la raillerie à la condescendance, les femmes ont été victimes d'un establishment critique largement dominé par les hommes. Les expérimentations formelles ne furent pas reconnues, là même où elles interrogeaient les " présupposés sexistes, voire misogynes [qui] détermin[ai]ent le sens de ce qu'on entend[ait] habituellement par le concept de thêâtralité» (37), et sur lesquels le répertoire est fondé, avec son lot d'intrigues et de motifs puisés à même la subordination des femmes dans l'espace social. Il s'agissait, donc, de «re-sémiotiser la scène» (47).

4 Dans une note de son article, Jane Moss évoque le même traitement réservé à «La marquise » (1970) de Marie Savard - texte publié sous le titre Bien à moi aux Éditions de la Pleine Lune en 1979, puis réédité avec une traduction de Louise Forsyth aux Éditions Trois en 1998. 5 Françoise Collin, «La lecture de l'illisible», Textuel $34 / 44, n^{\circ} 13,1984$, p. 7 . 
Jubinville s'attarde à mettre en évidence «les enjeux socioesthétiques et historiques que recouvre l'affaire des Fées» (60). Son étude fait par ailleurs voir que si le travail expérimental des femmes n'était pas pris en considération, on reconnaissait bien, pourtant, les révolutions formelles de ce que l'on appelait alors le «jeune théâtre» qui, jusque dans le processus de production du spectacle, faisait éclater les conventions établies. On sourit quand même à la lecture croisée des articles de Forsyth et de Jubinville, puisque dans son étude ce dernier cite Michel Bélair qui, en 1973, affirmait que la forme monologuée « représent[ait] un trait positif du "nouveau théâtre québécois" »(65). Apparemment, cela ne concernait que le théâtre écrit par des hommes...

Nutting s'arrête à un texte moins connu du canon féministe, Billy Strauss, de Lise Vaillancourt. Elle y scrute les rituels d'interaction, plus spécifiquement à travers trois dispositifs - la répétition, l'entretien et la conférence - qui, tous, organisent la parole dans une dynamique plaçant l'intersubjectivité à l'avant-plan. La notion de rituel souligne la nature déjà instituée de la forme, voire du contenu des énoncés. À travers ces dispositifs, la dramaturge soulignerait le poids des conventions - notamment «les comportements prévisibles que commandent les caractères typés» (84), en l'occurrence sexués - dans la construction du féminin.

Moss met en lumière la prégnance de la corporalité dans le théâtre des femmes, soulevant quelques-unes de ses dimensions : l'idéal de la beauté, l'anorexie et l'obésité, la grossesse et la maternité, l'érotisme tant lesbien qu'hétérosexuel, le viol et la ménopause (15). Ces différents aspects témoignent de la complexité de la question du corps, qui devient une obsession du questionnement féministe dans la mesure où le corps féminin est une obsession du social, et certainement le lieu fondamental de l'aliénation aux normes hétérosexistes.

Enfin, l'article de David invite avec bonheur à revisiter le corpus théâtral des femmes de cette période en envisageant la charge d'humour et de comique des textes féministes de la première heure. Dressant d'abord un bref historique, David s'attache à cerner un corpus à haut degré d'humour. Trois œuvres représentatives de la période retiennent son attention: Bernadette et Juliette ou La vie c'est comme la vaisselle, c'est toujours à recommencer (1978) d'Élizabeth Bourget, Moman (1979) de Louisette Dussault et Mademoiselle Autobody (1985) des Folles Alliées, troupe féministe basée à Québec. Selon lui, la présence du comique ne doit pas étonner, «si l'on veut bien admettre que le rire a toujours constitué une arme de choix dans les luttes des opprimés, quelles qu'aient été leurs causes » (100). Pourtant, on ne retient bien souvent que la dimension politique des textes de femmes, oubliant tout à fait à quel point, parfois, la charge ironique est forte: le comique n'est pas facilement reconnu aux femmes. L'auteur en convient lorsqu'il constate que

comparées aux succès à long terme remportés par les comédies qu'ont signées les auteurs masculins en synchronie, comme Broue (1979), Les voisins (1980), Durocher le milliardaire (1991) ou Matroni et moi (1995), les comédies au féminin, à quelques exceptions près, dont celle notable de Moman qui a franchi la barre des cinq cents représentations, n'ont pas, dans l'ensemble, connu un rayonnement aussi éclatant (102). 
L'écart est peut-être à mettre sur le compte du préjugé voulant «qu'il soit incompatible [...] d'être une femme et de vouloir faire rire» (102). La réponse à cet effacement se trouve probablement, souligne-t-il en fin d'article, du côté de la conjoncture, qui nous a fait passer d'un temps où le comique était «critique» à un comique strictement «ludique - juste pour rire -» (110; l'auteur souligne).

En complément de programme, pourrait-on dire - puisqu'il ne fait pas partie du dossier, étant présenté dans la rubrique «Document» - , un article de Lucie Robert sur Yvette Mercier-Gouin, dramaturge des années 1930 ayant l'insigne honneur d'être la première Québécoise - ici le féminin englobe le masculin - à avoir vu sa pièce, déjà « créée sur une scène professionnelle [...][]] être éditée sous la forme d'un livre par un éditeur indépendant» (117; l'auteure souligne), posant ainsi la première pierre, si l'on se réfère à la logique institutionnelle, à l'édifice du répertoire québécois. C'est pourtant Gratien Gélinas qui recevra le titre de «premier auteur dramatique d'importance dans l'histoire du Québec» (118; l'auteure souligne). Le troisième acte de la comédie dramatique de Mercier-Gouin, Cocktail, est publié à la suite de l'article de Robert.

Au-delà des contributions de chacun, ce dossier de L'annuaire théâtral souligne l'absence d'une étude de fond sur le corpus de textes dramaturgiques et sur les pratiques scéniques auxquels a donné lieu le mouvement des femmes. Car, comme le rappelle David, «la singularité et l'intensité des pratiques au féminin au cours des deux décennies concernées n'ont pas fait l'objet d'un réexamen à ce jour » (9), hormis deux numéros de la revue Jeu et un article ${ }^{6}$ paru dans Voix et Images. Il y a donc un réel « déficit de la réflexion historiographique et esthétique sur la production et la réception du théâtre des femmes de cette période» (9). De nombreuses études restent à venir, donc.

L'essai de Saint-Martin complète les investigations de la chercheuse, qui avait fait paraître auparavant Le nom de la mère. Mères, filles et écriture dans la littérature québécoise au féminin ${ }^{7}$. À l'heure où le modèle paternel est en redéfinition, mettant à mal ces "pères absents", essentiellement figures d'autorité, façonnés selon le breadwinner model, il est à propos de voir ce que la littérature en dit - et en fait. C'est donc aux «figures de pères qui dominent la fiction actuelle» (16) que Lori SaintMartin s'attarde. Et en abordant la question des pères et de la paternité, on aborde nécessairement celle des hommes et de la masculinité ainsi que celle des rapports de sexe et de genre, tout amalgamées qu'elles sont.

Or on le sait, les conceptions de la femme et du féminin ont été profondément bouleversées depuis les cinquante dernières années; les rapports homme-femme en

6 Jeannelle Laillou Savona, «La présence lesbienne dans le thêâtre féministe québécois des années 1975-1985 chez Marie-Claire Blais, Pol Pelletier et Jovette Marchessault», Voix et Images, vol. XXXVI, nº 1, automne 2010, p. 115-129. 7 Lori Saint-Martin, Le nom de la mère. Mères, filles et écriture dans la littérature québécoise au féminin, Québec, Nota bene, coll. «Essais critiques», 1999, 331 p. 
ont été transformés; la paternité s'en trouve également métamorphosée. C'est à ces transformations que s'intéresse Saint-Martin, s'attachant à en repérer les principaux motifs, de façon à «mesurer les effets littéraires des bouleversements sociaux survenus au cours des dernières décennies » (11). Du père incestueux au père aimant en quête de ses enfants, de nombreuses expressions de la paternité se font jour ici. Sans doute tous ces modèles existaient-ils aussi avant, mais ce qui a changé alors, ce sont les modalités du dire, de la prise de parole, et la possibilité des représentations; et cela, le livre de Saint-Martin le met clairement en lumière.

Examinant un corpus mixte d'une cinquantaine d'œuvres publiées depuis les trente dernières années, Saint-Martin distribue cette riche matière en cinq parties. Après une première partie posant les balises théoriques et les repères culturels nécessaires à la réflexion - où est énoncé d'emblée que "toute vision du père est une construction culturellement déterminée et susceptible de changer » (29) et où sont convoquées les notions d'autorité et de pouvoir étroitement liées à cette figure (Loi du Père, Nom du Père, etc.) —-, les deuxième et troisième sections montrent le père tel qu'il est évoqué par les enfants - puisque son statut est irrémédiablement intersubjectif ; c'est, encore plus que la mère, "l'enfant qui "fait" le père» (311). Certains lui font un procès, d'autres mènent une quête pour le (re)trouver - qu'il soit présent, absent, ou mort. La quatrième partie renverse la perspective : c'est du point de vue du père que la paternité est envisagée : tantôt refusée, tantôt empêchée, tantôt perdue... Enfin, la cinquième et dernière partie - à portée quasi rédemptrice, pourraiton dire - donne à voir les fictions que permettent les nouvelles paternités, qui ont pour effet de "réinventer la famille et le monde» ( $c^{\prime}$ est le titre du dernier chapitre). Mouvement d'ouverture bienvenu, porteur d'espoir, dans la mesure où une bonne partie des œuvres étudiées précédemment témoignaient de la lourdeur des conventions ayant enserré les hommes dans ce rôle contraignant.

À cet égard, bien que nombre de ces carcans aient éclaté, on se prend à constater l'absence d'œuvres de fiction mettant en scène l'homoparentalité, alors même que cette réalité se fait de plus en plus courante - elle donne lieu, à tout le moins, à de plus en plus d'essais. Il en est de même des réalités liées aux nouvelles technologies de reproduction, qui ont pourtant des échos au cinéma (le film Starbuck sur les écrans québécois à l'été 2011). La suite de la recherche se trouve peut-être de ce côté-là.

En définitive, ce qui a sans doute le plus changé au cours du dernier demisiècle, c'est l'abolition du clivage des rôles : mère-corps-affect contre père-esprit-sujet. Celle qui auparavant n'était «pas sujet et ne [pouvait] faire advenir la subjectivation de son enfant» (47) côtoie désormais un homme s'autorisant des rapports affectueux avec son enfant. "Nombre de textes [...] témoignent du désir d'une paternité dépouillée de ses connotations patriarcales, d'un corps-à-corps père-enfant sans fusion menaçante, d'une tendresse qui ne soit pas pour autant synonyme de faiblesse» (372), avance Saint-Martin parmi ses principales conclusions. Ainsi, « [l]'une des grandes nouveautés du corpus étudié, c'est précisément l'émergence de ces voix de pères qui formulent autrement la famille, la masculinité et les émotions » (382), en retrait du territoire de l'autorité distante, en quête de familiarité.

On se réjouit enfin de la prise en compte de plus en plus fréquente de corpus mixtes, ce qui a pour effet de montrer que les hommes ne sont pas tous porteurs de 
conceptions traditionnelles, pas plus que les femmes ne proposent toutes de nouvelles configurations imaginaires. Ultimement, le clivage - car il demeure - se déplace plutôt du côté des conceptions dont on se fait porteur, porteuse... peu importe le sexe.

Deuxième volet d'un diptyque sur la parentalité et surtout sur la charge symbolique accordée aux figures de la mère et du père, l'essai de Saint-Martin est foisonnant; aussi est-il difficile d'en parler en si peu de mots sans en réduire le propos: soulignons plutôt la grande concision des analyses, d'autant plus admirable quand on considère le nombre d'œuvres convoquées. Il en ressort que le père est un motif littéraire chargé de significations se situant au «carrefour d'interrogations et de visions différentes du pouvoir, de l'ordre social, des relations entre les sexes et les générations» (18-19). En plus de constats généraux - par exemple, que la paternité a le plus souvent été traitée depuis la perspective du fils alors qu'aujourd'hui la voix du père lui-même se fait entendre en littérature, et que ce changement de perspective «a des conséquences profondes» (17) -, l'étude relève des observations aussi fines que pertinentes pour mieux comprendre les changements de notre temps. 